Mathematical Modelling and Analysis

Volume 16 Number 1, March 2011, 119-142

Doi:10.3846/13926292.2011.562930

(C) Vilnius Gediminas Technical University, 2011
www.tandf.co.uk/journals/TMMA

Publisher: Taylor\&Francis and VGTU

Online ISSN: 1648-3510

Print ISSN: 1392-6292

\title{
A Numerical Method for a Stefan-Type Problem*
}

\author{
G. Shishkin ${ }^{a}$, L. Shishkina ${ }^{a}$, K. Cronin ${ }^{b}$, M. Stynes $^{b}$ and \\ M. Viscor ${ }^{b}$ \\ ${ }^{a}$ Institute of Mathematics and Mechanics, Russian Academy of Sciences \\ Ekaterinburg 620219, GSP-384, Russia \\ ${ }^{b}$ National University of Ireland Cork (UCC) \\ Cork, Ireland \\ E-mail: shishkin@imm.uran.ru \\ E-mail(corresp.): Lida@convex.ru \\ E-mail: k.cronin@ucc.ie; m.stynes@ucc.ie; m.viscor@ucc.ie
}

Received March 7, 2010; revised February 10, 2011; published online March 1, 2011

\begin{abstract}
A Stefan-type problem is considered. This is an initial-boundary value problem on a composite domain for a parabolic reaction-diffusion equation with a moving interface boundary. At the moving boundary between the two subdomains, an interface condition is prescribed for the solution of the problem and its derivatives. A finite difference scheme is constructed that approximates the initial-boundary value problem. An iterative Newton-type method for the solution of the difference scheme and a numerical method for the analysis of the errors of the computed discrete solutions are both developed.

Keywords: Stefan-type problem, initial-boundary value problem, composite domain, parabolic reaction-diffusion equation, moving interface boundary, finite difference scheme, iterative Newton-type method.
\end{abstract}

AMS Subject Classification: 80A22; 65M06; 65M15; 65M50; 49M15; 35K57.

\section{Introduction}

The drying of granular materials is a high intensity process that is widely used in the food and pharmaceutical industry, in chemical technology and in other manufacturing processes $[3,5]$. During the high temperature drying of granules in hot air flow, their heat and moisture undergo change through a wide range [4]. The distributions of moisture content and temperature, which depend

* This research was supported by the Russian Foundation for Basic Research under grant No. 10-01-00726, the Boole Centre for Research in Informatics at the National University of Ireland, Cork, and by the Mathematics Applications Consortium for Science and Industry (www.macsi.ul.ie) funded by the Science Foundation Ireland mathematics initiative grant 06/MI/005. 
on both the drying regime and material properties, define the kinetics of any associated chemical reactions [13].

The sharpest changes in the particle radius are observed under moisture evaporation during the boiling regime [12]. Consequently this stage is critical in the drying process and influences the properties of the final product. In the mathematical model, this drying stage is described by a Stefan-type problem; a description of a similar problem appears in [10]. In addition to the distributions of moisture content and temperature, one must find the position of the boiling front, i.e., the interface boundary between the dry and wet parts of the particle (the interface Stefan boundary) [12].

Stefan problems are nonlinear problems that lead to significant difficulties when constructing numerical methods for their solution; see, e.g., [1, 9] and its bibliography. One observes already that in the case of problems posed on composite domains with moving interface boundary — unlike problems with stationary interface boundaries - that the use of traditional grid approximations for the problem leads in general to large errors in the computed solutions (see, e.g., [11, Ch. 9]).

In the present paper we formulate a nonstandard Stefan-type mathematical problem with a moving interface boundary, and construct a finite difference scheme for its solution. When constructing the finite difference scheme, whenever a moving boundary is present we introduce a change of variables that transforms the original problem into a problem with stationary interface for which a grid approximation is constructed. On the temporal intervals where the interface boundary absents, the differential problem is approximated by a classical difference scheme (see, e.g., [9]).

The summary of the paper is the following. The formulation of the initialboundary value problem and the aims of our research are given in Section 2. To construct our finite difference scheme we use a form of domain decomposition. On the subdomain that includes the moving interface boundary, a suitable change of coordinates transforms the original problem into a problem with a stationary interface boundary but with moving lateral boundaries (Section 3). Then in these new variables, monotone grid approximations are constructed (Section 4). On that part of domain without any moving interface boundary, we construct a monotone grid approximation using the original variables. These approximations yield a difference scheme in the original variables (Section 5). Note that this difference scheme is nonlinear on the subdomain where a stationary interface boundary appears. A technique to analyze the convergence of the difference scheme, which is based on the discrete solution on a fine grid, is described in Section 6. An iterative method for solving this difference scheme is constructed in Section 7. For construction of a difference scheme when an interface boundary is present, one uses grid approximations of the differential problem written in the new variables (see Sections 3,4); some motivation for this approach is given in Section 8. In the same section the technique proposed in Section 6 for the analysis of convergence of our difference scheme is also discussed. 


\section{Problem Formulation. Aim of the Research}

We formulate a nonstandard Stefan-type problem in the case when the interface boundary between subdomains has points in common with the domain boundary. Consider the problem with the moving interface boundary on the set $\bar{G}$ where

$$
\bar{G}=\bar{G}_{(2.1)}=\bar{D} \times[0, T], \quad \bar{G}=G \cup S ; \quad S=S^{l} \cup S^{r} \cup S_{0} .
$$

Here $\bar{D}=[0,1], S$ is the domain boundary, $S^{l}, S^{r}$ and $S_{0}$ are the left, right and lower parts of the boundary $S$, and $S_{0}=\bar{S}_{0}$. Define the domains $G_{1}$ and $G_{2}$ by

$$
\begin{aligned}
& G_{1}=\{(x, t): 0<x<s(t), 0<t \leq T\}, \\
& G_{2}=\{(x, t): s(t)<x<1,0<t \leq T\},
\end{aligned}
$$

where $s(t)$ is a continuous function with $0 \leq s(t) \leq 1,0 \leq t \leq T$. Moreover,

$$
\begin{aligned}
& 0<s(t)<1 \quad \text { for } t_{1}<t<t_{2} ; \quad s\left(t_{1}\right)=1, \quad t \leq t_{1} ; \\
& s(t)=0, \quad t \geq t_{2} ; \quad 0<t_{1}, t_{2}<T .
\end{aligned}
$$

Thus, $\bar{G}_{1}=\emptyset, t>t_{2}, \bar{G}_{2}=\emptyset, t<t_{1}$. We have

$$
\bar{G}=\bar{G}_{1} \cup \bar{G}_{2}, \quad \bar{G}_{1} \cap \bar{G}_{2}=\bar{S}_{\text {int }} .
$$

Here

$$
\begin{aligned}
& \bar{G}_{i}=G_{i} \cup S_{i}, \quad S_{i}=S_{i}^{l} \cup S_{i}^{r} \cup S_{i t_{i-1}}, \quad i=1,2 ; \\
& S_{\text {int }}=\left\{(x, t): x=s(t), t_{1}<t \leq t_{2}\right\},
\end{aligned}
$$

the set $S_{\text {int }}$ is the interface boundary between the subdomains $G_{1}$ and $G_{2}$; $t_{0}=0, S_{1 t_{0}}=S_{10}=S_{0}, S_{2 t_{1}}=\left\{(x, t)=\left(1, t_{1}\right)\right\}$.

It is required to find the functions $s(t)$ for $t \in[0, T]$ and $u_{i}(x, t)$ for $(x, t) \in$ $\bar{G}_{i}$, where $i=1,2$, such that the following equations are satisfied:

$$
\begin{aligned}
& L_{i(2.2)} u_{i}(x, t)=0, \quad(x, t) \in G_{i}, \\
& u_{1}(x, t)=\varphi(x), \quad(x, t) \in S_{0}, \\
& \left.\begin{array}{l}
l^{l} u_{1}(x, t)=0, \quad 0<t<t_{2} \\
l^{l} u_{2}(x, t)=0, \quad t_{2}<t \leq T
\end{array}\right\}, \quad(x, t) \in S^{l}, \\
& \left.\begin{array}{l}
l^{r} u_{1}(x, t)=\psi(t), \quad 0<t<t_{1} \\
l^{r} u_{2}(x, t)=\psi(t), \quad t_{1}<t \leq T
\end{array}\right\}, \quad(x, t) \in S^{r}, \\
& \left.\begin{array}{rl}
l^{\text {int }}\left(u_{1}, u_{2} ; x, t\right)=0, & t_{1}<t<t_{2} \\
u_{1}(x, t)=u_{2}(x, t)=0, & t_{1} \leq t \leq t_{2}
\end{array}\right\}, \quad(x, t) \in \bar{S}_{\text {int }} ; \quad i=1,2 .
\end{aligned}
$$

Here

$$
\begin{aligned}
& L_{i(2.2)} u_{i}(x, t) \equiv a_{i}^{2} \frac{\partial^{2}}{\partial x^{2}} u_{i}(x, t)-\frac{\partial}{\partial t} u_{i}(x, t) \\
& l^{l} u_{i}(x, t) \equiv \frac{\partial}{\partial x} u_{i}(x, t), \quad l^{r} u_{i}(x, t) \equiv \frac{\partial}{\partial x} u_{i}(x, t)+u_{i}(x, t),
\end{aligned}
$$




$$
l^{i n t}\left(u_{1}, u_{2} ; x, t\right) \equiv \frac{\partial}{\partial x} u_{2}(x, t)-\frac{\partial}{\partial x} u_{1}(x, t) .
$$

The data of the problem (2.2), (2.1) are assumed to satisfy the condition

$$
\varphi(x)<0, \quad \frac{\partial}{\partial x} \varphi(x) \geq 0, \quad x \in[0,1], \quad \psi(t)>0, \quad 0 \leq t \leq T,
$$

and the functions $\varphi(x)$ and $\psi(t)$ are assumed to be sufficiently smooth.

By a solution of the Stefan-type problem (2.2), (2.1) we mean the function $s(t)$ (for $t \in[0, T]$ ) that defines the interface boundary $S_{\text {int }}$ and also the functions $u_{i}(x, t)$ (for $(x, t) \in \bar{G}_{i}$ ) with $i=1,2$ that satisfy the differential equations on the sets $G_{i}$, the boundary conditions on the set $S$ and the interface conditions on the interface boundary $S_{i n t}$. Note that the functions $u_{i}(x, t)$, $(x, t) \in \bar{G}_{i}$, which are continuous, satisfy the condition

$$
u_{1}(x, t) \leq 0, \quad(x, t) \in \bar{G}_{1}, \quad \text { and } \quad u_{2}(x, t) \geq 0, \quad(x, t) \in \bar{G}_{2} .
$$

Define the function $u(x, t),(x, t) \in \bar{G}$, to be equal to the functions $u_{i}(x, t)$ on the subsets $\bar{G}_{i}, i=1,2$, respectively. We say that $u$ is the solution of the Stefan-type problem.

The problem (2.2), (2.1) describes the high intensity drying process of wet granular materials in a hot air flow. At the advanced (main) stage of such a process, moisture is removed in the boiling regime, when the interior part of the particle is still wet (at a temperature not exceeding the boiling point) but the exterior part is already dry (at a temperature above boiling point). On the interface boundary, i.e., on the set $S_{i n t}$, the moisture concentration and temperature equal to zero and the boiling temperature, i.e., the corresponding functions $u_{1}(x, t)$ and $u_{2}(x, t)$ for $(x, t) \in S_{\text {int }}$ take prescribed values. Moreover, on the interface boundary the conservation law is fulfilled, i.e., the heat flow to the boundary caused by heat conduction is consumed for evaporation of moisture incoming to the boundary due to diffusion. Here flows of heat and moisture are defined by the derivatives $(\partial / \partial x) u_{2}(x+0, t)$ and $(\partial / \partial x) u_{1}(x-0, t)$, respectively. A physical model corresponding the problem $(2.2),(2.1),(2.3)$ is discussed also in 8.1 in Section 8.

Note that various types of such conditions are formulated in mathematical models describing flows in porous media (including the discontinuous solutions on interfaces); see, e.g., [2] in the case of the stationary interface boundary.

Our problem (2.2), (2.1) differs from the classical Stefan problem, where the interface condition also involves an additional term that is the product of the latent heat $H$ and the derivative $\frac{d}{d t} s(t)$. This difference makes our problem significantly more difficult to solve numerically.

We shall construct a numerical method for the solution of the problem (2.2), (2.1) that converges in the maximum norm.

Note that the solution of the problem (2.2), (2.1) has specific singularities that must be taken into account when constructing a robust numerical method. Most significant from them are following:

- Nonlinearities caused by the presence of a moving interface boundary (the boiling front) separating the wet and dry parts of the domain; 
- The complicated behaviour of the problem solution in a neighbourhood of the boiling front at the moments of the appearance and disappearance of this front - in particular the velocity of the moving boiling front becomes infinite at the moment of its disappearance.

Even nonlinearity of the problem leads to serious difficulties in constructing difference schemes convergent in the maximum norm and justification of their convergence. In the paper, numerical method for solving this problem is considered; theoretical justification of its convergence is not discussed. However, numerical experiments (see 8.3 in Section 8), and also analysis of convergence of the difference scheme (see Section 6) in the iterative numerical method for solving the nonlinear discrete problem (see Section 7) demonstrate convergence of the constructed method in the maximum norm with order of the convergence rate close to one in $x$ and $t$ (see discussions in 8.4 in Section 8).

\section{Reduction of the Problem (2.2), (2.1) to the Problem with the Stationary Interface Boundary for $t \in\left[t_{1}, t_{2}\right]$}

Construction of numerical methods is simplified in the case when boundaries between subdomains are stationary. In the case of the Stefan problem a finite difference scheme can be written out sufficiently simple in a neighbourhood of the interface boundary when this boundary is stationary.

On the set $\bar{G}$ for $t \in\left[t_{1}, t_{2}\right]$, i.e., on the set $^{1}$

$$
\bar{G}_{(3.1)}=\bar{G}_{\left[t_{1}, t_{2}\right]} \equiv \bar{G}_{(2.1)} \cap\left\{\left[t_{1}, t_{2}\right]\right\},
$$

we pass from the variables $x, t$ to the new variables $\xi, t$

$$
\xi=\xi(x, t)=a_{i}^{-1}(x-s(t)), \quad(x, t) \in \bar{G}_{i}, t \in\left[t_{1}, t_{2}\right], i=1,2,
$$

for which the interface boundary of subdomains becomes stationary [11]. In the new variables, the sets $\bar{G}_{i}$ for $t \in\left[t_{1}, t_{2}\right]$ transform into the sets $\widetilde{\widetilde{G}}_{i}$, the functions $u_{i}(x, t)$ transform into the functions $\widetilde{u}_{i}(\xi, t)$, and the boundary $x=$ $s(t)$ for $t_{1} \leq t \leq t_{2}$ transforms into the boundary $\xi=\xi(t)=0$ for $t_{1} \leq t \leq t_{2}$ the set $\bar{G}=\bar{G}_{(3.1)}$ transforms into the set $\overline{\widetilde{G}}$. As a result, we have

$$
\begin{array}{rlrl}
\widetilde{u}(\xi, t) & =u(x(\xi, t), t), & (\xi, t) & \in \overline{\widetilde{G}} \\
\widetilde{u}_{i}(\xi, t) & =u(x(\xi, t), t), \quad(\xi, t) \in \overline{\widetilde{G}}_{i}, \quad i=1,2 .
\end{array}
$$

Here

$$
\begin{aligned}
& \overline{\widetilde{G}}=\widetilde{G} \cup \widetilde{S}, \quad \overline{\widetilde{G}}=\overline{\widetilde{G}}_{1} \cup \overline{\widetilde{G}}_{2}, \quad \overline{\widetilde{G}}_{1} \cap \overline{\widetilde{G}}_{2}=\overline{\widetilde{S}}_{i n t}, \\
& \overline{\widetilde{G}}_{i}=\left\{(\xi, t): \xi=\xi(x, t), t \in\left[t_{1}, t_{2}\right], \quad(x, t) \in \bar{G}_{i}\right\}, \\
& \overline{\widetilde{G}}_{i}=\widetilde{G}_{i} \cup \widetilde{S}_{i}, \quad \widetilde{S}_{i}=\widetilde{S}_{i}^{l} \cup \widetilde{S}_{i}^{r} \cup \widetilde{S}_{i t_{1}}, \quad i=1,2 ; \\
& \widetilde{S}_{i n t}=\left\{(\xi, t): \xi=0, t_{1}<t \leq t_{2}\right\},
\end{aligned}
$$

${ }^{1}$ Here and further in the paper $\left\{\left[t_{*}, t^{*}\right]\right\} \equiv\left\{(x, t): x \in \bar{D}, t \in\left[t_{*}, t^{*}\right]\right\}, t_{*}, t^{*} \in[0, T]$. 
where $\left\{(\xi, t): \xi \in\left[-1 / a_{1}, 0\right], t=t_{1}\right\}, \widetilde{S}_{2 t_{1}}=\overline{\widetilde{S}}_{2 t_{1}}=\left\{(\xi, t)=\left(0, t_{1}\right)\right\},{\widetilde{S_{1}}}_{1}^{r}=$ $\overline{\widetilde{S}}_{2}^{l}=\overline{\widetilde{S}}_{\text {int }}$.

For $t \in\left[t_{1}, t_{2}\right]$ the problem $(2.2),(2.1)$ in the new variables transforms into the problem

$$
\begin{aligned}
& \widetilde{L}_{i(3.3)} \widetilde{u}_{i}(\xi, t)=0, \quad(\xi, t) \in \widetilde{G}_{i}, \\
& \left.\begin{array}{ll}
\widetilde{u}_{1}(\xi, t)=\widetilde{\varphi}_{t_{1}}(\xi), & \xi \in\left[-\frac{1}{a_{1}}, 0\right] \\
\widetilde{u}_{2}(\xi, t)=\widetilde{\varphi}_{t_{1}}(\xi), \quad \xi=0
\end{array}\right\}, \quad(\xi, t) \in \widetilde{S}_{t_{1}}, \\
& \widetilde{l}^{l} \widetilde{u}_{1}(\xi, t)=0, \quad t \in\left(t_{1}, t_{2}\right], \quad(\xi, t) \in \widetilde{S}_{1}^{l}, \\
& \widetilde{l}^{r} \widetilde{u}_{2}(\xi, t)=\psi(t), \quad t \in\left(t_{1}, t_{2}\right], \quad(\xi, t) \in \widetilde{S}_{2}^{r}, \\
& \left.\begin{array}{rl}
\widetilde{l}^{i n t}\left(\widetilde{u}_{1}, \widetilde{u}_{2} ; \xi, t\right) & =0, \quad t_{1}<t<t_{2} \\
\widetilde{u}_{1}(\xi, t)=\widetilde{u}_{2}(\xi, t) & =0, \quad t_{1} \leq t \leq t_{2}
\end{array}\right\}, \quad(\xi, t) \in \widetilde{\widetilde{S}}_{i n t} ; \quad i=1,2 .
\end{aligned}
$$

Here

$$
\begin{aligned}
& \widetilde{L}_{i(3.3)} \widetilde{u}_{i}(\xi, t) \equiv \frac{\partial^{2}}{\partial \xi^{2}} \widetilde{u}_{i}(\xi, t)+a_{i}^{-1} \frac{d}{d t} s(t) \frac{\partial}{\partial \xi} \widetilde{u}_{i}(\xi, t)-\frac{\partial}{\partial t} \widetilde{u}_{i}(\xi, t), \\
& \widetilde{l}^{l} \widetilde{u}_{1}(\xi, t) \equiv a_{1}^{-1} \frac{\partial}{\partial \xi} \widetilde{u}_{1}(\xi, t), \quad \widetilde{l}^{r} \widetilde{u}_{2}(\xi, t) \equiv a_{2}^{-1} \frac{\partial}{\partial \xi} \widetilde{u}_{2}(\xi, t)+\widetilde{u}_{2}(\xi, t), \\
& \widetilde{l}^{i n t}\left(\widetilde{u}_{1}, \widetilde{u}_{2} ; \xi, t\right) \equiv a_{2}^{-1} \frac{\partial}{\partial \xi} \widetilde{u}_{2}(\xi, t)-a_{1}^{-1} \frac{\partial}{\partial \xi} \widetilde{u}_{1}(\xi, t) ; \\
& \widetilde{\varphi}_{t_{1}}(\xi)=u_{1}\left(\xi\left(x, t_{1}\right), t_{1}-0\right), \quad\left(x, t_{1}\right) \in \bar{G}_{1} .
\end{aligned}
$$

It is obvious that the solution of the problem $(3.3),(3.2)$ is a pair $(\widetilde{u}(\xi, t), s(t))$. This problem has sufficiently simple conjunction conditions on the interface boundary, moreover, this interface boundary is stationary. But new problem has somewhat more complicated differential equations as compared with the problem $(2.2),(2.1)$ because convective terms appear in the equations. The lateral boundaries of the set $\overline{\widetilde{G}}$ become curvilinear.

\section{Difference Schemes on Subdomains for the Problem (2.2), (2.1)}

We construct a difference scheme for the problem $(2.2),(2.1)$ on the set $\bar{G}$, considering corresponding grid approximations of the problem on the temporal intervals $\left[0, t_{1}\right],\left[t_{1}, t_{2}\right]$ and $\left[t_{2}, T\right]$. Note that for $t \leq t_{1}$ and $t \geq t_{2}$ the problem $(2.2),(2.1)$ is a standard problem for a parabolic equation on a homogeneous domain.

4.1. We construct a grid approximation of the problem (2.2), (2.1) on the subset in $\bar{G}$ for $t \leq t_{1}$ :

$$
\bar{G}_{(4.1)}=\bar{G}_{\left[0, t_{1}\right]} \equiv \bar{G}_{(2.1)} \cap\left\{\left[0, t_{1}\right]\right\} .
$$


On the set $\bar{G}_{(2.1)}$ we introduce the rectangular (basic) grid

$$
\bar{G}_{h}=\bar{\omega} \times \bar{\omega}_{0}
$$

where $\bar{\omega}$ and $\bar{\omega}_{0}$ are meshes on the intervals $\bar{D}=[0,1]$ and $[0, T]$, respectively; the mesh size in $\bar{\omega}$ is $h$, the mesh size in $\bar{\omega}_{0}$ is $h_{0} ; h=N^{-1}, h_{0}=T N_{0}^{-1}$. Here $N+1$ and $N_{0}+1$ are the numbers of nodes in the meshes $\bar{\omega}$ and $\bar{\omega}_{0}$, respectively. Set $\bar{G}_{h}=G_{h} \cup S_{h}, S_{h}=S_{h}^{l} \cup S_{h}^{r} \cup S_{0 h}$, where $G_{h}=G \cap \bar{G}_{h}$, $S_{h}=S \cap \bar{G}_{h}, S_{h}^{l}, S_{h}^{r}$ and $S_{0 h}$ are the left, right and lower parts of the grid boundary, respectively.

We approximate the subproblem $(2.2),(2.1),(4.1)$ on the following grid in $\bar{G}_{h}$ for $t \in\left[0, t_{1}^{\tau}\right]$ :

$$
\begin{aligned}
& \bar{G}_{(1) h(4.3)}=\bar{G}_{h(4.2)} \cap\left\{\left[0, t_{1}^{\tau}\right]\right\}, \\
& \bar{G}_{(1) h}=G_{(1) h} \cup S_{(1) h}, \quad S_{(1) h}=S_{(1) h}^{l} \cup S_{(1) h}^{r} \cup S_{(1) 0 h},
\end{aligned}
$$

where the value $t_{1}^{\tau}$, which approximates the value $t_{1}$, is specified below.

When constructing difference schemes, we use monotone difference approximations of differential equations and boundary conditions [9]. For the problem $(2.2),(2.1)$ on the set $\bar{G}_{(4.1)}$ we consider the difference scheme on the grid $\bar{G}_{(1) h}=\bar{G}_{(1) h(4.3)}$ :

$$
\begin{aligned}
\Lambda_{1(4.4)} z_{1}(x, t) & =0, & & (x, t) \in G_{(1) h}, \\
z_{1}(x, t) & =\varphi(x), & & (x, t) \in S_{(1) 0 h}, \\
\lambda_{(4.4)}^{l} z_{1}(x, t) & =0, & & (x, t) \in S_{(1) h}^{l}, \\
\lambda_{(4.4)}^{r} z_{1}(x, t) & =\psi(t), & & (x, t) \in S_{(1) h}^{r} .
\end{aligned}
$$

Here

$$
\begin{aligned}
& \Lambda_{1(4.4)} z(x, t) \equiv a_{1}^{2} \delta_{\bar{x} \widehat{x}} z(x, t)-\delta_{\bar{t}} z(x, t), \\
& \lambda_{(4.4)}^{l} z(x, t) \equiv \delta_{x} z(x, t), \quad \lambda_{(4.4)}^{r} z(x, t) \equiv\left(\delta_{\bar{x}}+1\right) z(x, t) ;
\end{aligned}
$$

$\delta_{\bar{x} \widehat{x}} z(x, t)$ is the second-order difference derivative on a nonuniform grid, i.e.,

$$
\begin{aligned}
& \delta_{\bar{x} \widehat{x}} z(x, t)=z_{\bar{x} \widehat{x}}(x, t)=2\left(x^{i+1}-x^{i-1}\right)^{-1}\left[\delta_{x} z(x, t)-\delta_{\bar{x}} z(x, t)\right], \\
& (x, t)=\left(x^{i}, t\right) \in G_{(1) h}, \quad x^{i-1}, x^{i}, x^{i+1} \in \bar{\omega}
\end{aligned}
$$

$\delta_{x} z(x, t)$ and $\delta_{\bar{x}} z(x, t), \delta_{\bar{t}} z(x, t)$ are the first-order (forward and backward) difference derivatives, i.e.,

$$
\begin{array}{ll}
\delta_{x} z(x, t)=\left(x^{i+1}-x^{i}\right)^{-1}\left(z\left(x^{i+1}, t\right)-z\left(x^{i}, t\right)\right), & \left(x^{i}, t\right),\left(x^{i+1}, t\right) \in \bar{G}_{(1) h}, \\
\delta_{\bar{x}} z(x, t)=\left(x^{i}-x^{i-1}\right)^{-1}\left(z\left(x^{i}, t\right)-z\left(x^{i-1}, t\right)\right), & \left(x^{i-1}, t\right),\left(x^{i}, t\right) \in \bar{G}_{(1) h} \\
\delta_{\bar{t}} z(x, t)=\left(t^{j}-t^{j-1}\right)^{-1}\left(z\left(x^{i}, t^{j}\right)-z\left(x^{i}, t^{j-1}\right)\right), & \left(x, t^{j}\right),\left(x, t^{j-1}\right) \in \bar{G}_{(1) h}
\end{array}
$$

where $(x, t)=\left(x^{i}, t^{j}\right)$. The value $t_{1}^{\tau} \in \bar{\omega}_{0}$ that approximates $t_{1}$ is defined by the relations

$$
z_{1}(x, t) \leq 0, \quad t \leq t_{1}^{\tau}, \quad z_{1}(x, t)>0, \quad t>t_{1}^{\tau}, \quad t, t_{1}^{\tau} \in \bar{\omega}_{0}, \quad(x, t) \in \bar{S}_{h}^{r}
$$


The finite difference scheme for the problem (2.2), (2.1) for $t \leq t_{1}$, i.e., the scheme (4.4), (4.3), is constructed. On the set

$$
\bar{G}_{(1)}=\bar{G} \cap\left\{\left[0, t_{1}^{\tau}\right]\right\}
$$

using the function $z_{1}(x, t)$ for $(x, t) \in \bar{G}_{(1) h}$, we construct the bilinear interpolant

$$
\bar{z}_{1}(x, t), \quad(x, t) \in \bar{G}_{(1)},
$$

approximating the solution of the problem $(2.2),(2.1)$ on the set $\bar{G}_{(4.1)}$. Here and below, the first-order approximations are used to approximate boundary conditions because higher-order approximations cause difficulties for $t=t_{1}$ and $t=t_{2}$, i,e., at the moments of the appearance and disappearance of the moving interface, when, in general, $t_{i} \neq t_{i}^{\tau}$ for $i=1,2$, (see discussions in 8.3, 8.4 of Section 8).

4.2. Now we construct a grid approximation of the problem $(2.2),(2.1)$ for $t_{1} \leq t \leq t_{2}$, approximating the problem (3.3), (3.2) in the new variables $\xi, t$.

4.2.1. Preliminary we make some auxiliary constructs. Let a function $s^{\tau}(t)$, $t \in \bar{\omega}_{0}$ be a grid approximation of the function $s(t), t \in[0, T]$, satisfying the conditions

$$
\begin{aligned}
& 0 \leq s^{\tau}(t) \leq 1, \quad t \in \bar{\omega}_{0} ; \quad 0<s^{\tau}(t)<1, \quad t \in\left(t_{1}^{\tau}, t_{2}^{\tau}\right) \\
& s^{\tau}(t)=1, \quad t \in\left[0, t_{1}^{\tau}\right] ; \quad s^{\tau}(t)=0, \quad t \in\left[t_{2}^{\tau}, T\right] ; \quad t, t_{1}^{\tau}, t_{2}^{\tau} \in \bar{\omega}_{0} .
\end{aligned}
$$

We denote by $\bar{s}^{\tau}(t)$ for $t \in\left[t_{1}^{\tau}, t_{2}^{\tau}\right]$ the linear interpolant of the function $s^{\tau}(t)$ for $t \in \bar{\omega}_{0}^{\tau}$, where $\bar{\omega}_{0}^{\tau}=\left[t_{1}^{\tau}, t_{2}^{\tau}\right] \cap \bar{\omega}_{0}$. For the sets

$$
\bar{G}_{\left[t_{1}, t_{2}\right]}, \quad \bar{G}_{1\left[t_{1}, t_{2}\right]}, \quad \bar{G}_{2\left[t_{1}, t_{2}\right]},
$$

where $\bar{G}_{\left[t_{1}, t_{2}\right]}=\bar{G}_{\left[t_{1}, t_{2}\right](3.1)} \equiv \bar{G} \cap\left\{\left[t_{1}, t_{2}\right]\right\}, \bar{G}_{i\left[t_{1}, t_{2}\right]}=\bar{G}_{i} \cap\left\{\left[t_{1}, t_{2}\right]\right\}, i=1,2$, we associate the sets

$$
\bar{G}^{\tau}, \quad \bar{G}_{1}^{\tau}, \quad \bar{G}_{2}^{\tau},
$$

where $\bar{G}^{\tau}=\bar{G} \cap\left\{\left[t_{1}^{\tau}, t_{2}^{\tau}\right]\right\}, \bar{G}_{i}^{\tau}=\bar{G}_{i} \cap\left\{\left[t_{1}^{\tau}, t_{2}^{\tau}\right]\right\}, i=1,2$. We have

$$
\bar{G}^{\tau}=\bar{G}_{1}^{\tau} \cup \bar{G}_{2}^{\tau}, \quad \bar{G}_{1}^{\tau} \cap \bar{G}_{2}^{\tau}=\bar{S}_{i n t}^{\tau},
$$

where $\bar{G}^{\tau}=G^{\tau} \cup S^{\tau}, S^{\tau}=S^{\tau l} \cup S^{\tau r} \cup S_{t_{1}^{\tau}}^{\tau}, \bar{G}_{i}^{\tau}=G_{i}^{\tau} \cup S_{i}^{\tau}, S_{i}^{\tau}=S_{i}^{\tau l} \cup S_{i}^{\tau r} \cup$ $S_{i t_{1}^{\tau}}^{\tau}, i=1,2$, the set $S_{i n t}^{\tau}$ is the interface boundary between subdomains $G_{1}^{\tau}$ and $G_{2}^{\tau} ; S^{\tau l}, S^{\tau r}$ and $S_{t_{1}^{\tau}}^{\tau}$ are the left, right and lower parts of the boundary $S^{\tau}$, moreover, $S_{t_{1}^{\tau}}^{\tau}=\bar{S}_{t_{1}^{\tau}}^{\tau}$; in a similar way the boundary parts $S_{i}^{\tau}$ and $S_{2}^{\tau} t_{1}^{\tau}=$ $\left\{(x, t)=\left(1, t_{1}^{\tau}\right)\right\}$ are denoted. Here

$$
\begin{aligned}
& G_{1}^{\tau}=\left\{(x, t): 0<x<\bar{s}^{\tau}(t), t \in\left(t_{1}^{\tau}, t_{2}^{\tau}\right]\right\}, \\
& G_{2}^{\tau}=\left\{(x, t): \bar{s}^{\tau}(t)<x<1, t \in\left(t_{1}^{\tau}, t_{2}^{\tau}\right]\right\}, \\
& S_{\text {int }}^{\tau}=\left\{(x, t): x=\bar{s}^{\tau}(t), t \in\left(t_{1}^{\tau}, t_{2}^{\tau}\right]\right\} .
\end{aligned}
$$



where

On the set $\bar{G}^{\tau}$ we pass from the variables $x, t$ to the new variables $\xi, t$,

$$
\xi=\xi(x, t)=a_{i}^{-1}\left(x-\bar{s}^{\tau}(t)\right), \quad(x, t) \in \bar{G}_{i}^{\tau}, \quad t \in\left[t_{1}^{\tau}, t_{2}^{\tau}\right], \quad i=1,2,
$$

for which the interface boundary $S_{i n t}^{\tau}$ becomes stationary. In the new variables, the sets $\bar{G}_{i}^{\tau}$ transform into the sets $\overline{\widetilde{G}}_{i}^{\tau}$. We have

$$
\begin{aligned}
& \overline{\widetilde{G}}^{\tau}=\widetilde{G}^{\tau} \cup \widetilde{S}^{\tau}, \overline{\widetilde{G}}^{\tau}=\overline{\widetilde{G}}_{1}^{\tau} \cup \overline{\widetilde{G}}_{2}^{\tau}, \overline{\widetilde{G}}_{1}^{\tau} \cap \overline{\widetilde{G}}_{2}^{\tau}=\overline{\widetilde{S}}_{i n t}^{\tau} ; \overline{\widetilde{G}}_{i}^{\tau}=\widetilde{G}_{i}^{\tau} \cup \widetilde{S}_{i}^{\tau}, \\
& \overline{\widetilde{G}}_{i}^{\tau}=\left\{(\xi, t): \xi=\xi(x, t), t \in\left[t_{1}^{\tau}, t_{2}^{\tau}\right],(x, t) \in \bar{G}_{i}^{\tau}\right\}, \\
& \widetilde{S}_{i}^{\tau}=\widetilde{S}_{i}^{\tau} \cup \widetilde{S}_{i}^{\tau r} \cup \widetilde{S}_{i t}^{\tau}, \quad i=1,2 ; \quad \widetilde{S}_{i n t}^{\tau}=\left\{(\xi, t): \xi=0, t \in\left(t_{1}^{\tau}, t_{2}^{\tau}\right]\right\},
\end{aligned}
$$

where

$$
\begin{aligned}
& \widetilde{S}_{1 t_{1}^{\tau}}^{\tau}=\overline{\widetilde{S}}_{1 t_{1}^{\tau}}^{\tau}=\left\{(\xi, t): \xi \in\left[-a_{1}^{-1}, 0\right], t=t_{1}^{\tau}\right\} \\
& \widetilde{S}_{2 t_{1}^{\tau}}^{\tau}=\overline{\widetilde{S}}_{2 t_{1}^{\tau}}^{\tau}=\left\{(\xi, t)=\left(0, t_{1}^{\tau}\right)\right\} .
\end{aligned}
$$

4.2.2. We construct a difference scheme on the set $\overline{\widetilde{G}}_{(4.10)}^{\tau}$ in the following way. On the strip

$$
\overline{\widetilde{G}}^{\infty}=\left\{(\xi, t):-\infty<\xi<\infty, t \in\left[t_{1}^{\tau}, t_{2}^{\tau}\right]\right\}
$$

we introduce the uniform rectangular (basic) grid $\overline{\widetilde{G}}_{h}^{\infty}=\widetilde{\omega} \times \bar{\omega}_{0}^{\tau}, \overline{\widetilde{G}}_{h}^{\infty}=$ $\widetilde{G}_{h}^{\infty} \cup \widetilde{S}_{t_{1}^{\tau} h}^{\infty}$, where $\widetilde{\omega}$ and $\bar{\omega}_{0}^{\tau}$ are meshes on the $\xi$-axis and the interval $\left[t_{1}^{\tau}, t_{2}^{\tau}\right]$, respectively. The mesh size in $\widetilde{\omega}$ is $h_{\xi i}=N^{-1} / a_{i}, i=1,2$, where $N+1$ is the number of nodes in the mesh $\widetilde{\omega}$ per unit length on the $\xi$-axis. The point $\left(0, t_{1}^{\tau}\right)$ belongs to the grid $\overline{\widetilde{G}}_{h}^{\infty}$. On the set $\overline{\widetilde{G}}_{(4.10)}^{\tau}$ we construct the grid

$$
\overline{\widetilde{G}}_{h}^{\tau}=\widetilde{G}_{h}^{\tau} \cup \widetilde{S}_{h}^{\tau}
$$

Here the sets $\widetilde{G}_{h}^{\tau}$ and $\widetilde{S}_{h}^{\tau}$ are, respectively, the sets of the interior and boundary nodes in the grid $\overline{\widetilde{G}}_{h}^{\tau}$,

$$
\begin{aligned}
& \widetilde{G}_{h}^{\tau}=\widetilde{G}^{\tau} \cap \widetilde{\widetilde{G}}_{h}^{\infty}, \quad \widetilde{G}_{h}^{\tau}=\widetilde{G}_{1 h}^{\tau} \cup \widetilde{G}_{2 h}^{\tau} \cup \widetilde{S}_{i n t, h}^{\tau}, \\
& \widetilde{S}_{h}^{\tau}=\widetilde{S}_{h}^{\tau} \cup \widetilde{S}_{h}^{\tau} \cup \widetilde{S}_{t^{\tau} h}^{\tau}, \quad \widetilde{S}_{t^{\tau} h}^{\tau}=\widetilde{S}_{t_{1}^{\tau} h}^{\tau} \cup \widetilde{S}_{t^{\tau} h}^{\tau r} ;
\end{aligned}
$$

we set $\overline{\widetilde{S}}_{h}^{\tau r}=\widetilde{S}_{h}^{\tau r} \cup\left\{\left(0, t_{1}^{\tau}\right)\right\}$.

The sets $\widetilde{G}_{i h}^{\tau}$ and $\widetilde{S}_{i n t, h}^{\tau}$ are nodes in the grid $\widetilde{G}_{h}^{\tau}$, which belong to the sets $\widetilde{G}_{i}^{\tau}$ and $\widetilde{S}_{i n t}^{\tau}$, respectively, for $i=1,2$. Nodes of the sets $\widetilde{S}_{h}^{\tau l}$ and $\widetilde{S}_{h}^{\tau r}$ are generated by intersections of lines parallel to the $\xi$-axis that pass through nodes of the set $\widetilde{G}_{h}^{\tau}$, with the sets $\widetilde{S}^{\tau l}$ and $\widetilde{S}^{\tau r}$, respectively. Nodes of the sets $\widetilde{S}_{t_{1}^{\tau} h}^{\tau}$ and $\widetilde{S}_{t^{\tau} h}^{\tau r}$ are generated by intersections of lines parallel to the $t$-axis that pass through nodes of the set $\widetilde{G}_{h}^{\tau}$, with the sets $\widetilde{S}_{t_{1}^{\tau}}^{\tau}$ and $\widetilde{S}^{\tau r}$, respectively. 
Note that the grid $\overline{\widetilde{G}}_{h}^{\tau}$, as well as the set $\overline{\widetilde{G}}^{\tau}$, is defined by the function $s^{\tau}(t)$ for $t \in \bar{\omega}_{0}^{\tau}$, which is found (together with the grid solution approximating the solution of the differential problem) when solving the difference scheme.

Let $\widetilde{z}(\xi, t),(\xi, t) \in \overline{\widetilde{S}}_{h}^{\tau r}$ be some function. We denote by $\overline{\widetilde{z}}^{\tau r}(\xi, t)$, for $(\xi, t) \in \overline{\widetilde{S}}^{\tau r}$ the linear interpolant of this function constructed on the set $\overline{\widetilde{S}}^{\tau r}$.

We approximate differential problem (3.3), (3.2) by the following difference scheme on the grid $\overline{\widetilde{G}}_{h(4.11)}^{\tau}$ :

$$
\begin{aligned}
& \widetilde{\Lambda}_{i(4.12)} \widetilde{z}_{i}(\xi, t)=0, \quad(\xi, t) \in \widetilde{G}_{i h}^{\tau}, \quad i=1,2, \\
& \widetilde{z}_{1}(\xi, t)=\widetilde{z}_{1\{(4.4),(4.3)\}}(\xi, t), \quad(\xi, t) \in \widetilde{S}_{t_{1}^{\tau} h}^{\tau}, \\
& \widetilde{z}_{2}(\xi, t)=\widetilde{z}_{2}^{\tau r}(\xi, t), \quad(\xi, t) \in \widetilde{S}_{t^{\tau} h}^{\tau r}, \\
& \widetilde{\lambda}_{(4.12)}^{l} \widetilde{z}_{1}(\xi, t)=0, \quad t_{1}^{\tau}<t<t_{2}^{\tau}, \quad(\xi, t) \in \widetilde{S}_{h}^{\tau l}, \\
& \tilde{\lambda}_{(4.12)}^{r} \widetilde{z}_{2}(\xi, t)=\psi(\xi), \quad t_{1}^{\tau}<t \leq t_{2}^{\tau}, \quad(\xi, t) \in \widetilde{S}_{h}^{\tau r}, \\
& \left.\begin{array}{c}
\widetilde{\lambda}_{(4.12)}^{i n t}\left(\widetilde{z}_{1}, \widetilde{z}_{2} ; \xi, t\right)=0, \quad t_{1}^{\tau}<t<t_{2}^{\tau} \\
\widetilde{z}_{1}(\xi, t)=\widetilde{z}_{2}(\xi, t)=0, \quad t_{1}^{\tau} \leq t \leq t_{2}^{\tau}
\end{array}\right\}, \quad(\xi, t) \in \overline{\widetilde{S}}_{i n t, h}^{\tau} .
\end{aligned}
$$

Here

$$
\begin{aligned}
& \widetilde{\Lambda}_{i(4.12)} \widetilde{z}_{i}(\xi, t)=\widetilde{\Lambda}_{i(4.12)}\left(s_{h}(\cdot)\right) \widetilde{z}_{i}(\xi, t) \\
& \quad \equiv \delta_{\bar{\xi}} \widetilde{z}_{i}(\xi, t)+a_{i}^{-1} \delta_{\bar{t}} s_{h}(t) \delta_{\bar{\xi}} \widetilde{z}_{i}(\xi, t)-\delta_{\bar{t}} \widetilde{z}_{i}(\xi, t), \\
& \widetilde{\lambda}_{(4.12)}^{l} \widetilde{z}(\xi, t) \equiv a_{1}^{-1} \delta_{\xi} \widetilde{z}(\xi, t), \quad \widetilde{\lambda}_{(4.12)}^{r} \widetilde{z}(\xi, t) \equiv\left(a_{2}^{-1} \delta_{\bar{\xi}}+1\right) \widetilde{z}(\xi, t), \\
& \widetilde{\lambda}_{(4.12)}^{i n t}\left(\widetilde{z}_{1}, \widetilde{z}_{2} ; \xi, t\right) \equiv a_{2}^{-1} \delta_{\xi} \widetilde{z}_{2}(\xi, t)-a_{1}^{-1} \delta_{\bar{\xi}} \widetilde{z}_{1}(\xi, t) ;
\end{aligned}
$$

the functions $s_{h}(t)$ and $s^{\tau}(t)$ for $t \in \bar{\omega}_{0}^{\tau}$ in the formulae (4.8) and (4.12a) are subjected to the relations $s^{\tau}(\breve{t})=s_{h}(\breve{t})$ for $\breve{t} \leq t$ with $\breve{t}, t \in \bar{\omega}_{0}^{\tau}$; the function $s_{h}(t)$ is found when solving the difference scheme $(4.12 \mathrm{a}),(4.11) ; \delta_{\bar{\xi}} \widehat{\xi} \widetilde{z}(\xi, t)$ is the second-order difference derivative, in general, on a nonuniform grid,

$$
\begin{aligned}
& \delta_{\bar{\xi} \widehat{\xi}} \widetilde{z}(\xi, t)=\widetilde{z}_{\bar{\xi} \widehat{\xi}}(\xi, t)=2\left(\xi^{i+1}+\xi^{i-1}\right)^{-1}\left[\delta_{\xi} \widetilde{z}(\xi, t)-\delta_{\bar{\xi}} \widetilde{z}(\xi, t)\right], \quad(\xi, t) \in \widetilde{G}_{h}^{\tau}, \\
& \left(\xi^{i-1}, t\right),\left(\xi^{i}, t\right),\left(\xi^{i+1}, t\right) \in \overline{\widetilde{G}}_{h}^{\tau}, \quad(\xi, t)=\left(\xi^{i}, t\right),
\end{aligned}
$$

$\delta_{\xi} \widetilde{z}(\xi, t)$ and $\delta_{\bar{\xi}} \widetilde{z}(\xi, t), \delta_{\bar{t}} \widetilde{z}(\xi, t)$ are the first-order (forward and backward) difference derivatives,

$$
\begin{array}{cc}
\delta_{\xi} \widetilde{z}(\xi, t)=\left(\xi^{i+1}-\xi^{i}\right)^{-1}\left(\widetilde{z}\left(\xi^{i+1}, t\right)-\widetilde{z}\left(\xi^{i}, t\right)\right), & \left(\xi^{i}, t\right),\left(\xi^{i+1}, t\right) \in \overline{\widetilde{G}}_{h}^{\tau}, \\
\delta_{\bar{\xi}} \widetilde{z}(\xi, t)=\left(\xi^{i}-\xi^{i-1}\right)^{-1}\left(\widetilde{z}\left(\xi^{i}, t\right)-\widetilde{z}\left(\xi^{i-1}, t\right)\right), & \left(\xi^{i-1}, t\right),\left(\xi^{i}, t\right) \in \overline{\widetilde{G}}_{h}^{\tau}, \\
\delta_{\bar{t}} \widetilde{z}(\xi, t)=\left(t^{j}-t^{j-1}\right)^{-1}\left(\widetilde{z}\left(\xi, t^{j}\right)-\widetilde{z}\left(\xi, t^{j-1}\right)\right), & \left(\xi, t^{j}\right),\left(\xi, t^{j-1}\right) \in \overline{\widetilde{G}}_{h}^{\tau}, \\
(\xi, t)=\left(\xi^{i}, t^{j}\right), \quad t^{j}>t_{1}^{\tau} .
\end{array}
$$


The function $\varphi_{1}(\xi)$ is defined by the relation

$$
\varphi_{1}(\xi)=\bar{z}_{1}(x(\xi, t), t-0), \quad t=t_{1}^{\tau}, \quad(x, t) \in \bar{G}_{(1)},
$$

where $\bar{z}_{1}(x, t)=\bar{z}_{1(4.6)}(x, t), \bar{G}_{(1)}=\bar{G}_{(1)(4.5)}$.

The value $t_{2}^{\tau}$ is specified in the following way. Let for some $t^{*} \in \bar{\omega}_{0}$ with $t^{*}>t_{1}^{\tau}$, the solution of the difference scheme (4.12a), (4.11) exists under the condition $t<t^{*}$ and the function $s_{h}(t)$ satisfy the condition $s_{h}(t)>0$ for $t_{1}^{\tau}<t<t^{*}$. However, for $t=t^{*}$ the solution of the difference scheme (4.12a), (4.11), for which the condition $s_{h}\left(t^{*}\right)>0$ holds, does not exist (the conjunction condition of derivatives on $\widetilde{S}_{i n t, h}^{\tau}$ is violated for the discrete solution under the condition $\left.s_{h}\left(t^{*}\right)>0\right)$. In this case we set

$$
t_{2}^{\tau}=t^{*}
$$

and, moreover, we set $s_{h}\left(t_{2}^{\tau}\right)=0$, and we take the solution of the discrete problem (4.12a), (4.11), in which the conjunction condition of derivatives on $\widetilde{\widetilde{S}}_{i n t, h}^{\tau}$ is omitted, as the solution of the difference scheme for $t=t_{2}^{\tau}$. In nodes of the grid $\overline{\widetilde{G}}_{h(4.11)}^{\tau}$, where $t_{2}^{\tau}=t_{2(4.12 \mathrm{~b})}^{\tau}$, on the temporal level $t=t_{2}^{\tau}$ we have

$$
\begin{aligned}
& \widetilde{\Lambda}_{2(4.12)} \widetilde{z}_{2}(\xi, t)=0, \quad(\xi, t) \in \widetilde{G}_{2 h}^{\tau}, \\
& \widetilde{z}_{2}(\xi, \breve{t})=\overline{\widetilde{z}}_{2}^{\tau r}(\xi, \breve{t}), \quad(\xi, \breve{t}) \in \widetilde{S}_{t^{\tau} h}^{\tau r}, \quad \breve{t}<t, \\
& \tilde{\lambda}_{(4.12)}^{r} \widetilde{z}_{2}(\xi, t)=\psi(\xi), \quad(\xi, t) \in \widetilde{S}_{h}^{\tau r}, \\
& \widetilde{z}_{1}(\xi, t)=\widetilde{z}_{2}(\xi, t)=0, \quad(\xi, t) \in \overline{\widetilde{S}}_{i n t, h}^{\tau} ; \quad t=t_{2}^{\tau} \text {. }
\end{aligned}
$$

We call the function $\widetilde{z}_{i}(\xi, t)$ for $(\xi, t) \in \overline{\widetilde{G}}_{i h}^{\tau}$ with $i=1,2$, the solution of the difference scheme (4.12), (4.11); the function $s_{h}(t)$ for $t \in\left[t_{1}^{\tau}, t_{2}^{\tau}\right]$ defines the disposition of the interface boundary. The function $\widetilde{z}(\xi, t)$ for $(\xi, t) \in \overline{\widetilde{G}}_{h}^{\tau}$, such that $\widetilde{z}(\xi, t)=\widetilde{z}_{i}(\xi, t)$ for $(\xi, t) \in \widetilde{\widetilde{G}}_{i h}^{\tau}$ with $i=1,2$ is called also the solution of the difference scheme (4.12), (4.11).

Note that in the case when the function $s_{h}(t)$ for $t \in\left[t_{1}^{\tau}, t_{2}^{\tau}\right]$ is known, the difference scheme (4.12), (4.11), in which the conjunction condition of derivatives on $\overline{\widetilde{S}}_{i n t, h}^{\tau}$ is omitted, is monotone [9].

The difference scheme (4.12), (4.11) is nonlinear. For its solving, it is convenient to apply the iterative method given in [9] for nonlinear problems. When solving the difference scheme on each temporal step, the function $s_{h}(t)$ with $t \in \omega_{0}$ is computed in the iterative process so that to satisfy the conjunction conditions on $\widetilde{\widetilde{S}}_{\text {int, } h}^{\tau}$.

We construct an interpolant of the function $\widetilde{z}(\xi, t)$ for $(\xi, t) \in \overline{\widetilde{G}}_{h}^{\tau}$ on $\overline{\widetilde{G}}^{\tau}$. First, using the grid values $\widetilde{z}(\xi, t)$, we construct the function $\widetilde{z}^{\xi}(\xi, t)$ on the lines $t=$ const for $t \in \bar{\omega}_{0}^{\tau}$, which is the linear interpolant in $\xi$, extending it outside $\overline{\widetilde{G}} \tau$ as constants; between coordinate lines that pass through the nodes $\bar{\omega}_{0}^{\tau}$, we construct the linear interpolant in $t$ of the function $\widetilde{z}^{\xi}(\xi, t)$. We denote by $\overline{\widetilde{z}}(\xi, t)$ with $(\xi, t) \in \overline{\widetilde{G}}^{\tau}$ the function constructed. 
For $(\xi, t) \in \overline{\widetilde{G}}^{\tau}$ we return to the original variables $x, t$ :

$$
x=a_{i} \xi+\bar{s}_{h}(t), \quad(\xi, t) \in \overline{\widetilde{G}}_{i}^{\tau}, \quad i=1,2,
$$

where $\bar{s}_{h}(t)$ is an interpolant of the function $s_{h}(t)$. Under such transformation the set $\widetilde{\widetilde{G}}_{(4.10)}^{\tau}$ pass into the set $\overline{\widetilde{G}}^{\tau i n v}=\bar{G}_{(4.8)}^{\tau}$, and the function $\overline{\widetilde{z}}(\xi, t)$, $(\xi, t) \in \overline{\widetilde{G}} \tau$ transforms into the function

$$
\overline{\widetilde{z}}^{i n v}(x, t), \quad(x, t) \in \bar{G}^{\tau} .
$$

Note that in the variables $x, t$ the grid $\overline{\widetilde{G}}_{h(4.11)}^{\tau}$ is associated with the grid

$$
\overline{\widetilde{G}}_{h}^{\tau i n v},
$$

and the function $\widetilde{z}(\xi, t)$ for $(\xi, t) \in \overline{\widetilde{G}}_{h}^{\tau}$ is associated with the function

$$
\widetilde{z}^{i n v}(x, t), \quad(x, t) \in \overline{\widetilde{G}}_{h}^{\tau i n v} .
$$

4.3. We construct a grid approximation of the problem $(2.2),(2.1)$ on the set

$$
\bar{G}_{(4.16)}=\bar{G}_{\left[t_{2}, T\right]} \equiv \bar{G}_{(2.1)} \cap\left\{\left[t_{2}, T\right]\right\} .
$$

We approximate the subproblem (2.2), (2.1), (4.16) on the grid

$$
\begin{aligned}
& \bar{G}_{h(4.17)}=\bar{G}_{(2) h} \equiv \bar{G}_{h(4.2)} \cap\left\{\left[t_{2}^{\tau}, T\right]\right\}, \\
& \bar{G}_{(2) h(4.17)}=G_{(2) h} \cup S_{(2) h}, \quad S_{(2) h}=S_{(2) h}^{l} \cup S_{(2) h}^{r} \cup S_{(2) t_{2}^{\tau} h} .
\end{aligned}
$$

We associate this subproblem with the difference scheme on the grid $\bar{G}_{(2) h(4.17)}$

$$
\begin{aligned}
& \Lambda_{2(4.18)} z_{2}(x, t)=0, \quad(x, t) \in G_{(2) h}, \\
& z_{2}(x, t)=\varphi_{2}(x), \quad(x, t) \in S_{(2) t_{2}^{\tau} h}, \\
& \lambda_{(4.4)}^{l} z_{2}(x, t)=0, \quad(x, t) \in S_{(2) h}^{l}, \\
& \lambda_{(4.4)}^{r} z_{2}(x, t)=\psi(x), \quad(x, t) \in S_{(2) h}^{r} .
\end{aligned}
$$

Here

$$
\begin{aligned}
& \Lambda_{2(4.18)} z(x, t) \equiv a_{2}^{2} \delta_{\bar{x} \widehat{x}} z(x, t)-\delta_{\bar{t}} z(x, t), \\
& \varphi_{2}(x)=\overline{\widetilde{z}}_{(4.13)}^{i n v}(x, t-0), \quad(x, t) \in \bar{G}^{\tau}, \quad t=t_{2}^{\tau} .
\end{aligned}
$$

The finite difference scheme for the problem (2.2), (2.1) for $t \geq t_{2}^{\tau}$, i.e., the scheme (4.18), (4.17), is constructed.

On the set

$$
\bar{G}_{(2)(4.19)}=\bar{G} \cap\left\{\left[t_{2}^{\tau}, T\right]\right\}
$$

for the function $z_{2}(x, t)$ with $(x, t) \in \bar{G}_{(2) h}$ we construct the bilinear interpolant

$$
\bar{z}_{2}(x, t), \quad(x, t) \in \bar{G}_{(2)(4.19)} .
$$




\section{Difference Scheme for the Problem (2.2), (2.1)}

In Section 4 for the problem (2.2), (2.1) considered on subdomains, corresponding grid approximations are constructed. Subproblems on the sets $\bar{G}_{\left[0, t_{1}\right](4.1)}$ and $\bar{G}_{\left[t_{2}, T\right](4.16)}$ are approximated by the difference schemes (4.4), (4.3) and (4.18), (4.17), respectively. Using the change of variables, the subproblem on the set $\bar{G}_{\left[t_{1}, t_{2}\right](3.1)}$ is transformed into the subproblem (3.3), (3.2), which is approximated by the difference scheme (4.12), (4.11).

Thus, on the grid in $\bar{G}$ :

$$
\bar{G}_{h}^{*}= \begin{cases}\bar{G}_{(1) h(4.3)}, & (x, t) \in \bar{G}_{(1)(4.5)}, \\ \bar{G}_{h(4.14)}^{\tau i n v}, & (x, t) \in \bar{G}_{(4.8)}^{\tau}, \\ \bar{G}_{(2) h(4.17)}, & (x, t) \in \bar{G}_{(2)(4.19)},\end{cases}
$$

the following grid function is defined:

$$
z^{*}(x, t)= \begin{cases}z_{1(4.4)}(x, t), & (x, t) \in \bar{G}_{(1) h(4.3)}, \\ \widetilde{z}_{(4.15)}^{i n v}(x, t), & (x, t) \in \overline{\widetilde{G}}_{h(4.14)}^{i n v}, \\ z_{2(4.18)}(x, t), & (x, t) \in \bar{G}_{(2) h(4.17)} .\end{cases}
$$

We call the function $z^{*}(x, t)$ for $(x, t) \in \bar{G}_{h}^{*}$ the solution of the difference scheme $\{(4.4),(4.3) ;(4.12),(4.11) ;(4.18),(4.17)\}$.

Using the grid solutions, the interpolants $\bar{z}_{1(4.6)}(x, t)$ for $(x, t) \in \bar{G}_{(1)(4.5)}$, $\overline{\widetilde{z}}_{(4.13)}^{i n v}(x, t)$ for $(x, t) \in \bar{G}_{(4.8)}^{\tau}$ and $\bar{z}_{2(4.20)}(x, t)$ for $(x, t) \in \bar{G}_{(2)(4.19)}$. The interpolant

$$
\bar{z}^{*}(x, t)= \begin{cases}\bar{z}_{1(4.6)}(x, t), & (x, t) \in \bar{G}_{(1)(4.5)}, \\ \bar{z}_{(4.13)}^{i n v}(x, t), & (x, t) \in \bar{G}_{(4.8)}^{\tau}, \\ \bar{z}_{2(4.20)}(x, t), & (x, t) \in \bar{G}_{(2)(4.19)}\end{cases}
$$

approximates the solution of the problem (2.2), (2.1) on the whole set $\bar{G}$.

\section{Analysis of Errors to the Numerical Method for Prob- lem (2.2), (2.1) on the Basis of the Discrete Solution on the Finest Grid}

When analyzing the errors in the discrete solutions and also parameters characterizing convergence of the difference scheme, the technique is used similar to one given in $[6,7]$, however, it is modified taking into account peculiarities of the differential problem and the numerical method. When solving the difference scheme $\{(4.4),(4.3) ;(4.12),(4.11) ;(4.18),(4.17)\}$, we obtain the discrete solution $z_{(5.2)}^{*}(x, t)$ on the grid $\bar{G}_{h(5.1)}^{*}$. The function $z_{(5.2)}^{*}(x, t)$ is defined on the temporal levels $t=t^{j}$ on $\bar{\omega}_{0}$ in the nodes of the grid $\bar{G}_{h(5.1)}^{*}$, and the interpolant $\bar{z}_{(5.3)}^{*}(x, t)$ is defined on the whole set $\bar{G}$ and, in particular, on the temporal levels $t=t^{j}$ on $\bar{\omega}_{0}$ for $x \in[0,1]$. 
6.1. We expose a variant to compute the order of the convergence rate of the difference scheme on the family of the grids $\left\{\bar{G}_{h}^{* N^{*}}\right\}$ for $N^{*}=2^{i}$ with $i=5,6, \ldots, 10$, where $\bar{G}_{h}^{* N^{*}}$ is the grid $\bar{G}_{h(5.1)}^{*}$ for $N=N_{0}=N^{*}$. The interpolant $\bar{z}_{(5.3)}^{* N^{* F}}(x, t)$ is used as the exact solution of the differential problem $(2.2),(2.1)$. The interpolant is constructed using the discrete solution $z_{(5.2)}^{*}(x, t)$ computed on the finest grid $\bar{G}_{h}^{* N^{* F}}$ for $N^{*}=N^{* F}=2048$.

Errors in the numerical solutions in the maximum norm for each value $N^{*}$ are computed by the formula

$$
E^{N^{*}}=E^{N^{*}}\left(z^{* N^{*}}(\cdot)\right)=\left\|\bar{z}^{* N^{* F}}(x, t)-z^{* N^{*}}(x, t)\right\|_{\bar{G}_{h}^{* N^{*}}} .
$$

The values $E^{N^{*}}$ depending on $N^{*}$, which are represented in the form a table and/or a plot, allow us to come to qualitative conclusions on convergence of the solutions of the difference scheme and/or the order of their convergence rate.

The order of the convergence rate of the discrete solutions on the family of the grids $\left\{\bar{G}_{h}^{* N^{*}}\right\}$ (we say, the order of the local convergence rate) is defined by the formula

$$
q^{N^{*}}=\log _{2} \frac{E^{N^{*}}}{E^{2 N^{*}}} .
$$

The values $E^{N^{*}}$ and $E^{2 N^{*}}$ for the difference scheme are defined by the formula (6.1).

In the case when the value $q^{N^{*}}$ is fluctuating greatly as $N^{*}$ changes, it is necessary to compute the order of the local convergence rate by the formula

$$
q^{N^{*}, k}=k^{-1} \log _{2} \frac{E^{N^{*}}}{E^{2^{k} N^{*}}},
$$

where $k>1$ is even. The computations by formulae (6.1), (6.2) and (6.3) allow us to determine the value $q_{(x \& t)}$, i.e., the smallest order of the convergence rate in the variables $x$ and $t$. Set

$$
\begin{aligned}
& q_{(x \& t)}=\min _{N^{*}} q^{N^{*}} \quad \text { in the case of formula }(6.2), \\
& q_{(x \& t)}=\min _{N^{*}} q^{N^{*}, k} \quad \text { in the case of formula }(6.3) .
\end{aligned}
$$

From here, it follows the preliminary error bound for the discrete solution (the error estimate in the first approach):

$$
\left\|\bar{z}^{*\left(N^{F}, N_{0}^{F}\right)}-z^{*\left(N, N_{0}\right)}\right\|_{\bar{G}_{h}^{*\left(N, N_{0}\right)}} \leq M\left[N^{-q_{(x \& t)}}+N_{0}^{-q_{(x \& t)}}\right],
$$

where $M=M_{(6.4)}$, in general, is a sufficiently large constant.

6.2 . Now we expose a variant to compute orders of the convergence rate in $x$ and in $t$ for the difference scheme. The components of the errors due to discretization of the problem with respect to each of the variables $x$ and $t$ are defined in the following way. 
We denote by $\bar{G}_{h}^{*\left(N, N_{0}\right)}$ and $\bar{z}^{*\left(N, N_{0}\right)}(x, t)$ the grid $\bar{G}_{h(5.1)}^{*}$ and the solution $\bar{z}_{(5.3)}^{*}(x, t)$ of the discrete problem on this grid, respectively. Set

$$
E^{\left(N, N_{0}\right)}=E^{\left(N, N_{0}\right)}\left(z^{*\left(N, N_{0}\right)}(\cdot)\right)=\left\|\bar{z}^{*\left(N^{F}, N_{0}^{F}\right)}(x, t)-z^{*\left(N, N_{0}\right)}(x, t)\right\|_{\bar{G}_{h}^{*\left(N, N_{0}\right)}},
$$

where $\bar{z}^{*\left(N^{F}, N_{0}^{F}\right)}(x, t)$ is the solution on the finest grid $\bar{G}_{h}^{*\left(N^{F}, N_{0}^{F}\right)}$. Here $E^{\left(N, N_{0}\right)}$ is the error of the discrete solution $z^{*\left(N, N_{0}\right)}(x, t)$ on the fixed grid $\bar{G}_{h}^{*\left(N, N_{0}\right)}$.

The values $E^{\left(N, N_{0}^{F}\right)}$ and $E^{\left(N^{F}, N_{0}\right)}$ denote the components of the errors in $x$ and in $t$, respectively. The order of the local convergence rate of the discrete solutions in each variable in a neighbourhood of the solution $z^{*\left(N, N_{0}\right)}(x, t)$ for $(x, t) \in \bar{G}_{h}^{*\left(N, N_{0}\right)}$ is defined by the formulae:

$$
\begin{aligned}
& q^{\left(N, N_{0}^{F}\right)}=\log _{2} \frac{E^{\left(N, N_{0}^{F}\right)}}{E^{\left(2 N, N_{0}^{F}\right)}} \quad \text { for } x, \\
& q^{\left(N^{F}, N_{0}\right)}=\log _{2} \frac{E^{\left(N^{F}, N_{0}\right)}}{E^{\left(N^{F}, 2 N_{0}\right)}} \quad \text { for } t,
\end{aligned}
$$

where $2 N<N^{F}$ and $2 N_{0}<N_{0}^{F}$.

In the case when the value $q^{\left(N, N_{0}^{F}\right)}$ is fluctuating greatly as $N$ changes, it is necessary to compute the order of the local convergence rate in $x$ by the formula

$$
q^{\left(N, N_{0}^{F}\right), k}=k^{-1} \log _{2} \frac{E^{\left(N, N_{0}^{F}\right)}}{E^{\left(2^{k} N, N_{0}^{F}\right)}},
$$

where $2^{k} N<N^{F}$ and $k>1$ is even.

But if the value $q^{\left(N^{F}, N_{0}\right)}$ is fluctuating greatly as $N_{0}$ changes, it is necessary to compute the order of the local convergence rate in $t$ by the formula

$$
q^{\left(N^{F}, N_{0}\right), k}=k^{-1} \log _{2} \frac{E^{\left(N^{F}, N_{0}\right)}}{E^{\left(N^{F}, 2^{k} N_{0}\right)}},
$$

where $2^{k} N_{0}<N_{0}^{F}$ and $k>1$ is even. The values $q_{x}$ and $q_{t}$, i.e., orders of the convergence rate in $x$ and $t$, respectively, are defined by the relation

$$
q_{x}=\min _{N} q^{\left(N, N_{0}^{F}\right)}, \quad q_{t}=\min _{N_{0}} q^{\left(N^{F}, N_{0}\right)} .
$$

In the case when the values either $q_{(6.6)}^{\left(N, N_{0}^{F}\right)}$ or $q_{(6.7)}^{\left(N^{F}, N_{0}\right)}$ are fluctuating greatly as either $N$ or $N_{0}$ change, it is necessary to use in (6.10), respectively, the values $q_{(6.8)}^{\left(N, N_{0}^{F}\right), k}$ and $q_{(6.9)}^{\left(N^{F}, N_{0}\right), k}$.

6.3. Taking into account the orders of the convergence rate in $x$ and $t$, for the error of the discrete solution we obtain the estimate

$$
\left\|\bar{z}^{*\left(N^{F}, N_{0}^{F}\right)}-z^{*\left(N, N_{0}\right)}\right\|_{\bar{G}_{h}^{*\left(N, N_{0}\right)}} \leq M\left[N^{-q_{x}}+N_{0}^{-q_{t}}\right],
$$

where $M_{(6.11)}$ is a sufficiently large constant. 
Define more exactly the constant $M_{(6.11)}$. Taking into account the relations

$$
E^{\left(N, N_{0}^{F}\right)}=M^{\left(N, N_{0}^{F}\right)} N^{-q_{x}}, \quad E^{\left(N^{F}, N_{0}\right)}=M^{\left(N^{F}, N_{0}\right)} N^{-q_{x}},
$$

we find the values $M^{\left(N, N_{0}^{F}\right)}$ and $M^{\left(N^{F}, N_{0}\right)}$. Set

$$
M_{x}=\max _{N} M^{\left(N, N_{0}^{F}\right)}, \quad M_{t}=\max _{N_{0}} M^{\left(N^{F}, N_{0}\right)} .
$$

Thus, for the error of the discrete solution one has

$$
\left\|\bar{z}^{*\left(N^{F}, N_{0}^{F}\right)}-z^{*\left(N, N_{0}\right)}\right\|_{\bar{G}_{h}^{*\left(N, N_{0}\right)}} \leq M\left[N^{-q_{x}}+N_{0}^{-q_{t}}\right],
$$

where $M_{(6.14)}=\max \left[M_{x}, M_{t}\right]$. We call the error estimate (6.14) the realistic error bound because it is more reliable than (6.4).

The given technique of numerical analysis of the parameters characterizing convergence of the difference scheme $\{(4.4),(4.3) ;(4.12),(4.11) ;(4.18),(4.17)\}$ allows us to find orders of the convergence rate in $x$ and $t$ of the solution to the difference scheme and also error bounds of the discrete solution. The estimate (6.14), which is a posteriori, allows us to point out the values $N$ and $N_{0}$, under which the solution of the discrete problem has the required accuracy.

\section{Iterative Numerical Method for Solving the Discrete Problem (4.12), (4.11)}

Consider an iterative numerical method for solving the discrete problem (4.12), (4.11) where $t^{j} \in\left(t_{1}^{\tau}, t_{2}^{\tau}\right)$. Here we assume that the problem (4.12), (4.11) has already been solved for $t \leq t^{j-1}$, the function $s_{h}(t)$ is known for $t_{1}^{\tau} \leq t<t^{j}$, and the grid $\widetilde{\widetilde{G}}_{h(4.11)}^{\tau} \cap\left\{t \leq t^{j-1}\right\}$ has been constructed. It is required to find the value $s_{h}\left(t^{j}\right)$ and the functions $\widetilde{z}_{i}\left(\xi, t^{j}\right)$ for $i=1,2$.

7.1. We transform the problem $(4.12),(4.11)$ to a form that is suitable for iterative computation. If at the level $t=t^{j}$ one takes some value, say $\widehat{s}$, instead of the value $s_{h}\left(t^{j}\right)$, then it is possible to solve the problem (4.12a), in which case the interface condition for derivatives on the interface boundary $\widetilde{S}_{i n t, h}^{\tau}$ is omitted. Thus in this case, the interface condition on derivatives in general is not fulfilled by the computed solution. In the iterative process, we will choose the value $\widehat{s}$ in such a way that the discrete solution should satisfy the interface condition on the derivatives.

Note that the value $s_{h}\left(t^{j}\right)$ defines the variable $\xi$ (see (4.9)) and the grid $\overline{\widetilde{G}}_{h(4.11)}^{\tau}$ at the level $t=t^{j}$. Let $\widehat{s}$ be an arbitrary value in $D$. When constructing the spatial grid at $t=t^{j}$, one chooses $\widehat{s}$, instead of the value $s_{h}\left(t^{j}\right)$, such that

$$
s_{h}\left(t^{j}\right)=\widehat{s} .
$$

Hence, the discrete value $\xi$ is defined at the level $t=t^{j}$ as

$$
\xi=\xi(x, t)=a_{i}^{-1}(x-\widehat{s}), \quad(\xi, t) \in \overline{\widehat{\widetilde{G}}}_{i h}^{\tau} \cap\left\{t=t^{j}\right\}, \quad i=1,2,
$$


and the grid for $t \leq t^{j}$ is defined as

$$
\left\{\overline{\widetilde{G}}_{h(4.11)}^{\tau} \cap\left\{t \leq t^{j-1}\right\}\right\} \cup\left\{\overline{\widetilde{\widetilde{G}}}_{1 h}^{\tau} \cap\left\{t=t^{j}\right\}\right\} \cup\left\{\overline{\widetilde{G}}_{2 h}^{\tau} \cap\left\{t=t^{j}\right\}\right\} .
$$

For $t \leq t^{j}$ this grid is

$$
\overline{\widetilde{G}}_{h}^{\tau}=\widehat{\widetilde{G}}_{h}^{\tau} \cup \widehat{\widetilde{S}}_{h}^{\tau}
$$

here $\widehat{\widetilde{G}}_{h}^{\tau}$ and $\widehat{\widetilde{S}}_{h}^{\tau}$ are, respectively, the sets of interior and boundary nodes in the grid $\overline{\widetilde{G}}_{h}^{\tau}$. Similar notations are used for discrete subsets in the problem (4.12) at $t \leq t^{j}$.

Let $\widehat{\widetilde{z}}(\xi, t)$ be the solution of the following discrete problem at the time level $t=t^{j}$ for $(\xi, t) \in \overline{\widehat{\widetilde{G}}}_{h}^{\tau} \cap\left\{t=t^{j-1}, t^{j}\right\}:$

$$
\begin{aligned}
\widetilde{\Lambda}_{i(4.12)} \widehat{\widetilde{z}}_{i}(\xi, t) & =0, & (\xi, t) & \in \widehat{\widetilde{G}}_{i h}^{\tau} \cap\left\{t=t^{j}\right\}, \quad i=1,2, \\
\widehat{\widetilde{z}}_{1}(\xi, t) & =\widetilde{z}_{1}(\xi, t), & (\xi, t) & \in \widehat{\widetilde{S}}_{t_{1}^{\tau} h}^{\tau} \cap\left\{t=t^{j-1}\right\}, \\
\widehat{\widetilde{z}}_{2}(\xi, t) & =\widetilde{z}_{2}(\xi, t), & (\xi, t) & \in \widehat{\widetilde{S}}_{t^{\tau} h}^{\tau r} \cap\left\{t=t^{j-1}\right\}, \\
\widetilde{\lambda}_{(4.12)}^{l} \widehat{\widetilde{z}}_{1}(\xi, t) & =0, & (\xi, t) & \in \widehat{\widetilde{S}}_{h}^{\tau l} \cap\left\{t=t^{j}\right\}, \\
\widetilde{\lambda}_{(4.12)}^{r} \widehat{\widetilde{z}}_{2}(\xi, t) & =\psi(\xi), & (\xi, t) & \in \widehat{\widetilde{S}}_{h}^{\tau r} \cap\left\{t=t^{j}\right\}, \\
\widehat{\widetilde{z}}_{1}(\xi, t)= & \widehat{\widetilde{z}}_{2}(\xi, t)=0, & (\xi, t) & \in \overline{\widetilde{\widetilde{S}}}_{i n t, h}^{\tau} \cap\left\{t=t^{j}\right\} .
\end{aligned}
$$

Define the function $q(\widehat{s})$ by setting

$$
q(\widehat{s})=a_{2}^{-1} \delta_{\xi} \widehat{\widetilde{z}}_{2}(\xi, t)-a_{1}^{-1} \delta_{\bar{\xi}} \widehat{\widetilde{z}}_{1}(\xi, t), \quad \xi=\xi_{(7.1 \mathrm{~b})}\left(\widehat{s}, t^{j}\right)=0, \quad t=t^{j}
$$

We now discuss its attributes. The properties of the solution of problem (2.2), (2.1), (2.3) show that the function $q(\widehat{s})$ satisfies the condition $q\left(s_{h}\left(t^{j-1}\right)\right)>0$. Moreover, in a small neighbourhood of the value $\widehat{s}=s_{h}\left(t^{j-1}\right)$, the function $q(\widehat{s})$ decreases as $\widehat{s}$ decreases.

Such a behaviour of the function $q(\widehat{s})$ can be explained in the following way. According to the experimental results in Figs. 1 and 2 (see 8.3 in Section 8), the function $\bar{z}(x, t)$ grows as $x$ and/or $t$ increase (see Fig. 2 for $\bar{z}(x, t)$ ), and the function $\bar{s}^{\tau}(t)$ decreases when $t$ grows (see Fig. 2 for $\bar{s}^{\tau}(t)$ ). For $x=\bar{s}^{\tau}\left(t^{j}\right)$, $t^{j} \in\left(t_{1}^{\tau}, t_{2}^{\tau}\right)$ we have $\delta_{x} \bar{z}(x, t)-\delta_{\bar{x}} \bar{z}(x, t)=0$ (see Fig. 2 for $\left.\bar{z}(x, t)\right)$. Let for $t=$ $t^{j-1}, t^{j}$ the condition $\bar{s}^{\tau}\left(t^{j}\right)=\bar{s}^{\tau}\left(t^{j-1}\right)$ be fulfilled. Then, taking into account the behaviour of the function $\bar{z}\left(x, t^{j-1}\right)$, we obtain $\delta_{x} \bar{z}(x, t)-\delta_{\bar{x}} \bar{z}(x, t)>0$, $x=\bar{s}^{\tau}(t), t=t^{j}$, that gives $q\left(s_{h}\left(t^{j-1}\right)\right)>0$. In a similar way we verify that $q(\widehat{s})$ in a neighbourhood of $\widehat{s}=s_{h}\left(t^{j-1}\right)$ decreases as $\widehat{s}$ decreases.

For some value $\widehat{s}=\widehat{s}^{0}$, where $0<\widehat{s}^{0} \leq s_{h}\left(t^{j-1}\right)$, one may have

$$
q\left(\widehat{s}^{0}\right)=0 \text { and } q(\widehat{s})>0 \text { for } \widehat{s}^{0}<\widehat{s} \leq s_{h}\left(t^{j-1}\right)
$$

In this case we set

$$
s_{h}\left(t^{j}\right)=\widehat{s}^{0} .
$$


Note that when $\widehat{s}$ decreases further, the function $q(\widehat{s})$ can take positive values. But if

$$
q(\widehat{s})>0 \text { for } 0<\widehat{s} \leq s_{h}\left(t^{j-1}\right)
$$

then we set

$$
s_{h}\left(t^{j}\right)=0
$$

in this case the value $t_{2}^{\tau}$ is defined by the relation

$$
t_{2}^{\tau}=t^{j}
$$

Thus the relations (7.4)a,b,c define the values $s_{h}\left(t^{j}\right)$ and $t_{2}^{\tau}$. Consequently, at the time level $t=t^{j}$ we have the grid

$$
\overline{\widetilde{G}}_{h}^{\tau}=\overline{\widehat{\widetilde{G}}}_{h}^{\tau} \text { for } \widehat{s}=s_{h(7.4)}\left(t^{j}\right), \quad t=t^{j} .
$$

Define the function $\widetilde{z}_{(7.5)}\left(\xi, t^{j}\right)$ by

$$
\widetilde{z}(\xi, t)=\widehat{\widetilde{z}}(\xi, t), \quad(\xi, t) \in \overline{\widetilde{G}}_{h}^{\tau} \cap\left\{t=t^{j}\right\} .
$$

We call the function

$$
\widetilde{z}_{(7.5)}(\xi, t), \quad(\xi, t) \in \overline{\widetilde{G}}_{h(7.5)}^{\tau} \cap\left\{t=t^{j}\right\}
$$

the solution of the problem (7.2), (7.1), (7.3) for $t=t^{j}$. The value $s_{h(7.4)}\left(t^{j}\right)$ gives us the position of the interface boundary. One has

$$
\widetilde{z}_{(7.5)}(\xi, t)=\widetilde{z}_{\{(4.12),(4.11)\}}(\xi, t), \quad(\xi, t) \in \overline{\widetilde{G}}_{h(7.5)}^{\tau} \cap\left\{t=t^{j}\right\},
$$

i.e., the problems (7.2), (7.1), (7.3) and (4.12), (4.11) are equivalent for $t=t^{j}$.

7.2. We give an iterative method for solving the discrete problem (7.2), (7.1), (7.3) for $t^{j} \in\left(t_{1}^{\tau}, t_{2}^{\tau}\right]$; we seek an approximation of the value $\widehat{s}^{0}$ that is the root of the equation $q(\widehat{s})=0$ satisfying the condition (7.3a).

7.2.1. First, consider an approximation algorithm of the value $\widehat{s}^{0}$ in the case when the following condition is satisfied:

$$
t^{j-1}>t_{1}^{\tau}
$$

As preliminary approximations of $\widehat{s}^{0}$, choose values $\widehat{s}_{-1}$ and $\widehat{s}_{0}$ that help us to compute an value $\widehat{s}_{1}$ that is the first approximation of $\widehat{s}^{0}$. We call the values $\widehat{s}_{-1}$ and $\widehat{s}_{0}$ preliminary parameters. Set

$$
\widehat{s}_{-1}=s_{h}\left(t^{j-2}\right), \quad \widehat{s}_{0}=s_{h}\left(t^{j-1}\right) .
$$

Compute $q\left(\widehat{s}_{-1}\right), q\left(\widehat{s}_{0}\right)$; note that $q\left(\widehat{s}_{-1}\right), q\left(\widehat{s}_{0}\right)>0$. The value $\widehat{s}_{1}$ is the root of the equation

$$
\frac{\widehat{s}_{0}-\widehat{s}_{1}}{q\left(\widehat{s}_{0}\right)}=\frac{\widehat{s}_{-1}-\widehat{s}_{0}}{q\left(\widehat{s}_{-1}\right)-q\left(\widehat{s}_{0}\right)} .
$$


Now we find the value $q\left(\widehat{s}_{1}\right)$. Refinement of the approximations of $\widehat{s}^{0}$ is achieved as follows: for three sequential values

$$
\widehat{s}_{i-2}, \widehat{s}_{i-1}, \widehat{s}_{i}
$$

let the values

$$
q\left(\widehat{s}_{i-2}\right), q\left(\widehat{s}_{i-1}\right), q\left(\widehat{s}_{i}\right)
$$

be found, and among them let $q\left(\widehat{s}_{i-1}\right)$ and $q\left(\widehat{s}_{i}\right)$ be the smallest in absolute values. The improved approximation $\widehat{s}_{i+1}$ is defined to be the root of the equation

$$
\frac{\widehat{s}_{i}-\widehat{s}_{i+1}}{q\left(\widehat{s}_{i}\right)}=\frac{\widehat{s}_{i-1}-\widehat{s}_{i}}{q\left(\widehat{s}_{i-1}\right)-q\left(\widehat{s}_{i}\right)} .
$$

Then compute $q\left(\widehat{s}_{i+1}\right)$. The improvement process terminates when $i=I$, where $I$ is defined to be the smallest $i$ such that

$$
\left|\widehat{s}_{i}-\widehat{s}_{i-1}\right| \leq \delta
$$

and $\delta=\delta\left(N, N_{0}\right)$ is some small number. Set

$$
\widehat{s}^{0}=\widehat{s}_{I}
$$

7.2.2. Let now the following condition be satisfied

$$
t^{j-1}=t_{1}^{\tau}
$$

In this case, the values $\widehat{s}_{-1}$ and $\widehat{s}_{0}$ are taken as preliminary parameters for approximations of the value $\widehat{s}^{0}$, and the value $\widehat{s}_{1}$ is taken as the first approximation. The values $\widehat{s}_{-1}, \widehat{s}_{0}$ and $\widehat{s}_{1}$ are given below. Set

$$
\widehat{s}_{-1}=1 \text {. }
$$

We compute the value $q\left(\widehat{s}_{-1}\right)$ in the following way. Let $z_{1}(x, t)$ be the solution of the problem (4.4a), (4.3) for $t \leq t^{j}$ (the condition (4.4b) is omitted). Then $q\left(\widehat{s}_{-1}\right)$ is computed by

$$
q\left(\widehat{s}_{-1}\right)=a_{2}^{-1}\left[\psi\left(t^{j}\right)-z_{1}\left(1, t^{j}\right)\right]-a_{1}^{-1} \delta_{\bar{x}} z_{1}\left(1, t^{j}\right) .
$$

We have $q\left(\widehat{s}_{-1}\right)>0$. Prescribe the value

$$
\widehat{s}_{0}=1-h,
$$

where $h$ is the step-size in the grid $\bar{G}_{h(4.3)}$ on the $x$-axis, and compute the value $q_{h(7.2 \mathrm{~b})}\left(\widehat{s}_{0}\right)$.

Choose the first approximation $\widehat{s}_{1}$ to be the root of the equation

$$
\frac{\widehat{s}_{0}-\widehat{s}_{1}}{q\left(\widehat{s}_{0}\right)}=\frac{\widehat{s}_{-1}-\widehat{s}_{0}}{q\left(\widehat{s}_{-1}\right)-q\left(\widehat{s}_{0}\right)},
$$

then compute $q\left(\widehat{s}_{1}\right)$. Thus for $\widehat{s}_{-1}, \widehat{s}_{0}, \widehat{s}_{1}$ we obtain the related values $q\left(\widehat{s}_{-1}\right)$, $q\left(\widehat{s}_{0}\right), q\left(\widehat{s}_{1}\right)$. 
This iterative process is continued by following the procedure (7.8), (7.9).

7.2.3. We call the collection of the procedures defined by the relations $\{(7.6),(7.7) ;(7.10),(7.11) ;(7.8),(7.9)\}$ the iterative algorithm for the computation of the value $\widehat{s}^{0}$ based on the solution of the problem (7.2), (7.1), (7.3), which is equivalent to the problem (4.12), (4.11).

This Newton-type iterative algorithm allows us to find the solution of the discrete problem (4.12), (4.11); the computational accuracy in the iterative process is defined by the value $\delta_{(7.9)}$.

\section{Motivation of our Approach to the Construction of the Mathematical Model, the Numerical Method to Solve the Problem and Analyze Its Convergence}

This section discusses the formulation of the mathematical problem, the numerical method used to solve it, and the technique used to analyze its convergence.

8.1. The drying of wet materials in the case of "plane" geometry (see, e.g., $[12])$ is defined by the distributions of temperature $T(x, t)$ and moisture content $W(x, t)$. Under sufficiently simple conditions, i.e., under constant parameters in the heat and diffusion processes, the dimensionless temperature and moisture content obey heat conduction equations with constant heat and diffusion Fourier numbers, respectively. In this case the functions $T(x, t), W(x, t)$ and $u(x, t)$ satisfy the following "physical" relations

$$
\begin{array}{lll}
u_{2}(x, t)=T(x, t)-T_{\text {boil }}, & W(x, t)=0 & \text { for } T(x, t)>T_{\text {boil }}, \\
u_{1}(x, t)=-W(x, t), & T(x, t)=T_{\text {boil }} & \text { for } W(x, t)>0,
\end{array}
$$

where $T_{\text {boil }}$ is the boiling temperature.

The functions $s(t)$ and $\vartheta(t)$ for $t_{1} \leq t \leq t_{2}$, where $\vartheta(t)$ defines the boiling front $x=\vartheta(t)$, satisfy $s(t)=\vartheta(t)$; on the boiling front one has

$$
T(x, t)=T_{\text {boil }}, \quad W(x, t)=0 \quad \text { for } x=\vartheta(t) .
$$

The position of the boiling front is defined by the first-order derivatives of the solution from the left and right at the boiling front (up to a factor, which is the latent heat, that may depend on the solution).

The boundary condition on the left domain boundary corresponds to a symmetry condition for the heat and diffusion processes at the body centre and, on the right domain boundary, to conditions of heat-/ and mass-/transfer of the body within a medium according to Newton's law.

8.2. Note that the diffusion coefficients in a material with small moisture content are several orders of magnitude smaller than the heat conduction coefficients; see, e.g., [8]. This induces a moving interior layer for $0<s(t)<1$. Difficulties in the approximation of solutions (and their derivatives) in the case of singularly perturbed problems with moving boundary and interior layers are discussed in [11]; the errors in the discrete solutions computed by standard numerical methods can be of the same order of magnitude as the solutions 
themselves, and errors in the discrete derivatives of the solutions can be many times greater than the derivatives them selves. In Stefan-type problems, errors in the position of the boiling-water front, which is found in the solving problem process, can lead to large errors in the solution and its derivatives in a neighbourhood of that front.

In the method for solving problems with moving layers that is developed in [11], a special grid approximation of the problem is constructed in a new coordinate system in which the moving layer becomes stationary. This allows us to approximate in the maximum norm both the solution and its first-order derivatives in the spatial variables. The same approximation is applied in the present paper to approximate the problem (2.2), (2.1) for $t_{1} \leq t \leq t_{2}$; see Sections 3, 4.

8.3. The velocity of the moving boiling front becomes unbounded as the width of the wet part of the material becomes small. This causes significant difficulties when using iterative methods to solve the nonlinear Stefan problem.

Fig. 1 displays the computed values of $\bar{z}_{1}(x, t)=\overline{\widetilde{z}}_{1}^{i n v}(x, t)$ and $\bar{z}_{2}(x, t)=$ $\overline{\widetilde{z}}_{2}^{i n v}(x, t)$. These are the components of the function $\overline{\bar{z}}_{(4.13)}^{i n v}(x, t)$ for $(x, t) \in$ $\overline{\widetilde{G}}_{(4.8)}^{\tau}$.

Fig. 2 shows cross-sections of the function $\bar{z}(x, t)$ for $0 \leq t \leq 0.8$ at various times and the position of the interface boundary $\bar{S}_{\text {int (4.8) }}^{\tau}$ for $t_{1}^{\bar{\tau}} \leq t \leq t_{2}^{\tau}$.

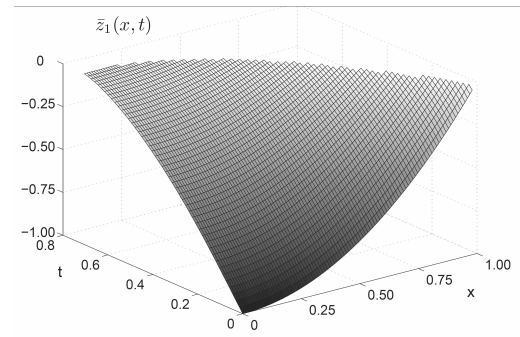

(a)

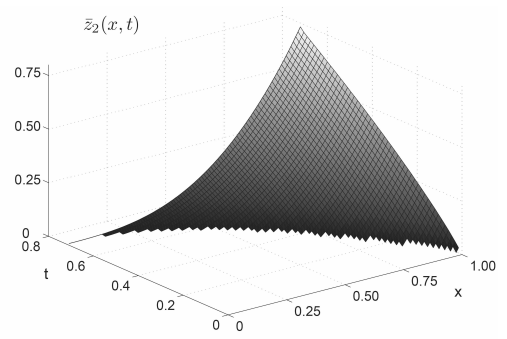

(b)

Figure 1. The functions $\bar{z}_{1}(x, t)$ and $\bar{z}_{2}(x, t)$ for $\boldsymbol{a}_{1}=\mathbf{1 . 0}$ and $\boldsymbol{a}_{2}=\mathbf{0 . 5}$

The number of iterations required to solve the Stefan problem when using the Newton-type method (Section 7 ) is typically about 6 , and only for $t \approx t_{2}^{\tau}$ does it increase to 9 . We observe that the number of iterations increases very slowly with the numbers of nodes.

Using the standard iterative bisection method on the part of the domain for $\xi<0, t_{1}^{\tau}<t<t_{2}^{\tau}$, the number of iterations is around $20-25$.

It should be note that for $t$ close to $t_{2}^{\tau}$ the moving velocity of the interface boundary becomes sufficiently large (see the curve on Fig. 2). Such a behaviour of the interface boundary could lead to large errors in the numerical solution. However, when approaching to $t_{2}^{\tau}$, the derivatives in $x$ of the function $\bar{z}(x, t)$ in a neighbourhood of the curve $\overline{\widetilde{S}}_{i n t}^{\tau}$ and on the set $\bar{G}_{1}^{\tau}$ become sufficiently small (see the function $\bar{z}(x, t)$ in Fig. 2) that leads to decrease of error in the numerical solution. As a result of summarized errors in both the approximation 


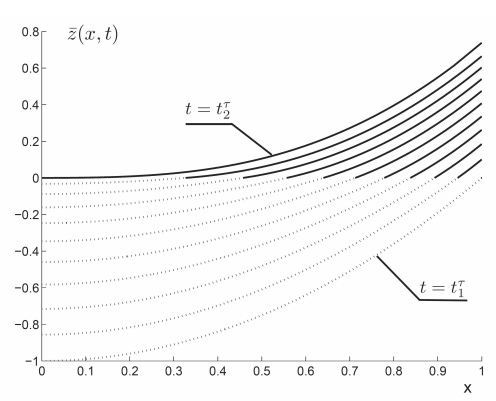

(a)

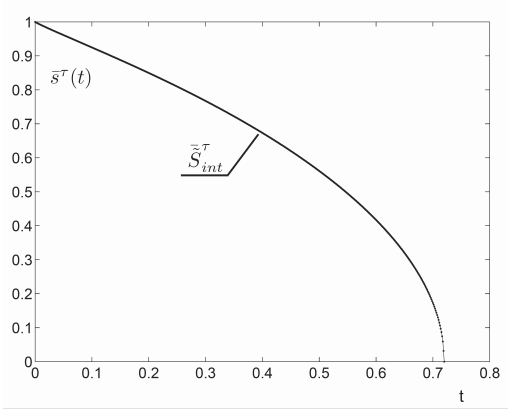

(b)

Figure 2. Cross-sections of the function $\bar{z}(x, t)$ for $t_{1}^{\tau} \leq t \leq t_{2}^{\tau}$ at various times (a) and the position of the interface boundary $\overline{\widetilde{S}}_{\text {int }}^{\tau}$ (b); $N^{*}=512, t_{1}^{\tau}=0, t_{2}^{\tau}=0.72$

of problem $(2.2),(2.1)$ and the Newton-type iterative method, for the discrete solution we obtain an experimental order of the convergence rate close to one (see 8.4 in Section 8).

8.4. Some comments on the convergence analysis of the numerical method. To study experimentally the convergence rate of numerical methods, researchers often use the "double mesh technique" [6] (where one uses the solution computed after bisecting the current mesh) or the computed solution on the finest grid available [6]). This approach is sufficiently accurate but only under the condition that the problem solution is sufficiently smooth so that it is possible to expand the discrete solution in terms of the step-size in the spatial and temporal variables. But our Stefan problem does not have the required smoothness: in general, $u \notin C^{2,1}$ in a neighbourhood of the points $\left(1, t_{1}\right)$ and $\left(0, t_{2}\right)$.

In general, while $\tau_{1}, \tau_{2} \in \bar{\omega}_{0}$, one does not have $t_{1}, t_{2} \in \bar{\omega}_{0}$. For this reason when solving a discrete problem on grids with constant step-sizes in space and time, the values $\tau_{1}, \tau_{2}$, and also the grid solutions can change somewhat erratically as the step size is reduced. A formal application of the technique from [6] leads to difficulties in the analysis of convergence of the difference scheme. On the other hand, the technique from Section 6 allows us to confirm the convergence of the difference scheme in the maximum norm and to find its convergence rate. Thus for example, when $a_{1}=1, a_{2}=0.5$, numerical experiments using the formulae (6.2) and (6.3) yield $q^{N^{*}}=0.73, q^{N^{*}, 4}=0.85$ with $N^{*}=2048$, i.e., the difference scheme (4.4), (4.3); (4.12), (4.11); (4.18), (4.17) converges. From the formulae (6.8), (6.9) and (6.13) with $N^{F}=N_{0}^{F}=$ 2048 we find $q^{\left(N, N_{0}^{F}\right), 4}=0.82, q^{\left(N^{F}, N_{0}\right), 4}=0.84$ and $M_{x}=0.70, M_{t}=2.89$.

Thus for the solution of the difference scheme we have the estimate (6.14), where $M=2.89, q_{x}=0.82, q_{t}=0.84$; the scheme converges with order close to one in $x$ and $t$. 


\section{Conclusion}

1. For the model Stefan-type problem, a finite difference scheme is constructed that uses classical approximations of the differential equation; when constructing the scheme, the problem on the part of the domain that includes the moving interface boundary is transformed to a problem with a stationary interface boundary.

2. An iterative Newton-type method is constructed to solve the nonlinear problem that approximates the problem in the presence of the moving interface boundary.

3. A technique of numerical analysis of convergence to the discrete solution is suggested that uses the solutions of discrete problems on the finest grids.

4. Numerical experiments show the usefulness of the difference scheme and of the method for the analysis of its convergence. The scheme converges with order of the convergence rate close to one.

5. The physical appropriateness of the parameters and equations in the mathematical model allows us to adapt it to the modelling of real drying processes.

\section{Acknowledgement}

The authors are grateful to the participants of the 14th International Conference MMA2009 (Daugavpils, Latvia, 2009) for interesting and helpful discussions of the research results.

\section{References}

[1] B.M. Budak, F.P. Vasiliev and A.B. Uspenskii. Difference method for solving of some boundary value problem of stefan type. Numerical methods in gas dynamics. Publ. of MSU, Moscow, issue 4, 4:139-183, 1965. (in Russian)

[2] R. Čiegis and A. Zemitis. The mathematical simulation of the liquid transport in multilayered nonwoven. Mathematical modelling and analysis, 2:9-34, 1997.

[3] A.A. Dolinsky. High-temperature spray drying. Drying Technology, 19(5):785806, 2001. Doi:10.1081/DRT-100103770.

[4] A.A. Dolinsky, K.D. Maletskaya and V.V. Shmorgun. Kinetics and Technology of Spray Drying. Naukova Dumka, Kiev, 1987. (in Russian)

[5] V.V. Farid. The moving boundary problems from melting and freezing to drying and frying of food. Chemical Engineering and Processing, 41:1-10, 2002. Doi:10.1016/S0255-2701(00)00153-7.

[6] P.A. Farrell, A.F. Hegarty, J.J.H. Miller, E. O'Riordan and G.I. Shishkin. Robust Computational Techniques for Boundary Layers. Chapman and Hall/CRC Press, Boca Raton, 2000. 
[7] S. Li, G.I. Shishkin and L.P Shishkina. Approximation of the solution and its derivative for the singularly perturbed black-scholes equation with nonsmooth initial data. Comp. Maths. Math. Phys., 47(3):442-462, 2007. Doi:10.1134/S0965542507030098.

[8] A.V. Luikov. Heat and Mass Transfer. Mir Publishers, Moscow, 1980.

[9] A.A. Samarskii. The Theory of Difference Schemes. Marcel Dekker, New York, 2001.

[10] G.I. Shishkin. On a problem of stefan type with discontinuous moving boundary. Soviet Math. Dokl., 16(5):1409-1412, 1975.

[11] G.I. Shishkin and L.P Shishkina. Difference Methods for Singular Perturbation Problems. Monographs \& Surveys in Pure \& Applied Math. Chapman and Hall/CRC, 2009.

[12] G.I. Shishkin, L.P Shishkina, K. Cronin, M. Cronin Stynes and M. Viscor. Heatmass-transfer for granular drying; mathematical modelling. In Mathematical and Informational Technologies in Management, Engineering and Education, Proceedings of the 3-d International Scientific Conference, pp. 16-23. Ekaterinburg: UGTU/UPI, 2009. (in Russian)

[13] R.T. Toledo. Fundamentals of Food Process Engeneering. third ed. Springer Science+Business Media, LLC, New York, 2007. 